Gut, 1976, 17, 993-997

\title{
Potential difference across the normal and the abnormal gastric mucosa in man
}

\author{
A. HOSSENBOCUS ${ }^{1}$, P. FITZPATRICK ${ }^{2}$, AND D. G. COLIN-JONES \\ From Southampton University Medical School, Southampton
}

SUMMARY The mucosal potential difference in the body of the stomach was measured in 18 patients with gastric ulcers, five with gastritis, and three with pernicious anaemia. The results were compared with those of 12 normal volunteers. Significantly lower than normal levels were found in the groups of patients with gastric ulcers and gastritis. The patients with pernicious anaemia had even lower potential differences. In an additional 25 subjects, measurements were made in the duodenal bulb and at five sites in the stomach under direct vision at endoscopy. Biopsies were taken from each site for histology. Subjects with gastritis at the high lesser curve had a significantly lower potential difference at that site than those without gastritis. In the latter group, the potential difference at the high lesser curve was as low as in the antrum, and invariably lower than at the high greater curve.

Exposure to eugenol of the gastric mucosa of dogs with Heidenhain pouches leads to an increase in the back-diffusion of hydrogen ions, and to apparent hyposecretion after stimulation with histamine (Davenport et al., 1964). The human gastric mucosa responds similarly to a number of agents, including aspirin (Smith et al., 1971) and bile salts (Ivey et al., 1970). Davenport (1965) postulated that the abnormal gastric mucosa of patients with gastric ulcers allowed excessive reabsorption of secreted hydrogen ions, thereby causing apparent hyposecretion. Permeability studies in gastric ulcer patients have provided some evidence in favour of this hypothesis (Chapman et al., 1968; Chapman et al., 1972; Ivey and Clifton 1974). However, problems with recovery of instilled solutions, neutralisation, secretion, and gastric emptying make such studies difficult in man, and the results are sometimes inaccurate.

Agents that impair the gastric mucosal barrier in man also lower the gastric mucosal potential difference (Geall et al., 1970; Hossenbocus and Colin-Jones, 1975). Studies in animals have shown a correlation between the increased sodium and hydrogen ion fluxes, and the fall in the potential difference (Davenport et al., 1964; Chvasta and Cooke, 1972). Since measurement of the potential

\footnotetext{
1Present address: Princess Alexandra Hospital, Woolloongabba, Brisbane, Queensland, Australia.

2Present address: Queen Alexandra Hospital, Portsmouth, Hants.

Received for publication 26 July 1976
}

difference does not suffer from the drawbacks associated with permeability studies, it was used for comparing the abnormal gastric mucosa of patients with gastric ulcers, gastritis, and pernicious anaemia with the normal. In an additional group of patients requiring diagnostic endoscopy, measurements were made and biopsies taken from specific sites under direct vision in order to obtain more direct correlation between histology and potential difference.

\section{Methods}

\section{CONVENTIONAL TECHNIQUE}

Potential difference was measured by the method of Anderson and Grossman (1965). The reference electrode for intravenous insertion consisted of a fine polyethylene tube (internal diameter $1.14 \mathrm{~mm}$ ) containing $3 \%$ agar saturated with potassium chloride. This was sterilised by gamma radiation. The intragastric electrode was a similar tube (internal diameter $3 \mathrm{~mm}$ ) containing the same material. Potential difference was measured with balanced calomel half-cells (K-100 Radiometer, Copenhagen) and recorded on paper (Servograph Pen Drive REA 310, Radiometer, Copenhagen) via a pH meter (Titrator TTT 2, Radiometer, Copenhagen).

After an overnight fast, the subject was kept in the sitting position while the exploring electrode was passed orally to about $80 \mathrm{~cm}$. The subject then lay on his left side, and the reference electrode was introduced into a peripheral vein via a sterile 
Table 1 Highest gastric mucosal potential difference recorded in four normal subjects on two separate occasions

\begin{tabular}{lll}
\hline Subject & \multicolumn{2}{l}{ Measurement of $P D(-m V)$} \\
\cline { 2 - 3 } & 1 & 2 \\
\hline 1 & 45 & 45 \\
2 & 49 & 44 \\
3 & 42 & 43 \\
4 & 43 & 43 \\
\hline
\end{tabular}

needle. The circuit was completed, and the exploring electrode was withdrawn $5 \mathrm{~cm}$ at a time while potential difference was recorded continuously. The highest potential difference was obtained when the tip of the electrode was $45-50 \mathrm{~cm}$ from the incisor teeth. This figure was accepted only if it was steady for at least 10 minutes. With this technique there was no need to use fluoroscopy to position the exploring electrode. The results of a preliminary study in which the potential difference was measured on two separate occasions in four normal subjects are shown in Table 1.

The subjects studied consisted of 12 normal volunteers, 18 patients with benign lesser curve gastric ulcers, five with atrophic gastritis, and three with treated pernicious anaemia. All the patients with gastric ulcers or gastritis had the diagnosis confirmed with endoscopy and multiple biopsies.

\section{MEASUREMENT UNDER DIRECT VISION}

For measurement of the potential difference at endoscopy, the conventional technique was modified by replacing the exploring electrode with a finer polyethylene tube (internal diameter $1.4 \mathrm{~mm}$ ) containing the same material. This was threaded through the biopsy channel of the GIF D2 (Olympus) endoscope.

After an overnight fast, the patient was premedicated with intravenous diazepam in a dose of 20-30 mg. Atropine was not used. The reference electrode was inserted in an antecubital vein in the left arm. During endoscopy, which was carried out with the subject lying on his left side, a note was made as to whether there was bile-stained fluid in the stomach. Fluid was sucked out before passing the exploring electrode through the biopsy channel. The tip was pressed gently against the mucosa, and the potential difference was recorded for one minute. Measurements were made in the duodenal bulb, the prepyloric region, at the incisura, the low greater curve, the high lesser curve, and the high greater curve. Biopsies were then taken from each one of those sites.

Twenty-five subjects needing diagnostic endoscopy were studied. Technically satisfactory tracings were obtained in 22 . The endoscopic diagnosis for those was: gastric ulcer (five), duodenal ulcer (four), pyloric ulcer (two), hiatus hernia (one), no lesion seen (10).

\section{HISTOLOGY}

Biopsy specimens were fixed in buffered formol saline and processed routinely. Sections were stained with haematoxylin and eosin and examined by one of us (P.F.) without any knowledge of the potential differences recorded. The recommendations of Whitehead were followed (Whitehead et al., 1972; Whitehead, 1973).

Informed consent was obtained from all 63 subjects studied. The safety of the subject from electrical hazards was ensured by following the recommendations in the Hospital Technical Memorandum 8 (Department of Health and Social Security, 1969).

\section{Results}

CONVENTIONAL TECHNIQUE

The group of 12 normal subjects had a potential difference of $-45.7 \pm 0.6 \mathrm{mV}$. (mean $\pm \mathrm{SEM}$ ) in the body of the stomach. As shown in Table 2, this

Table 2 Gastric mucosal potential difference in body of stomach in normal subjects

\begin{tabular}{lcc}
\hline Investigators & Subjects (no.) & $\begin{array}{l}P D(-m V) \\
\text { Mean } \pm 5 \text { EM }\end{array}$ \\
\hline Andersson and & 5 & $43 \cdot 6 \pm 4 \cdot 7$ \\
Grossman & & $\vdots$ \\
Geall et al. & 9 & $35 \cdot 5 \pm 0.5$ \\
Bennett et al. & 5 & $53.8 \pm 2 \cdot 3$ \\
Hossenbocus and & 12 & $45 \cdot 7 \pm 0.6$ \\
Colin-Jones & & \\
\hline
\end{tabular}

is similar to the findings of Andersson and Grossman (1965) and Bennett et al. (1974), but somewhat higher than those of Geall et al. (1970).

The results for all four groups in this study are shown in Fig. 1. The patients with gastric ulcers had a lower potential difference $(-32.4 \pm 0.7 \mathrm{mV})$ than the normal subjects. Statistical analysis using the $t$ test showed the difference between the means to be significan: $(\mathrm{P}<0.001)$.

The mean for the patients with gastritis, $-33.6 \mathrm{mV}$, was also significantly lower $(P<0.01)$ than that for the normal group.

The group of patients with pernicious anaemia was too small for statistical analysis. However, all three patients had potential differences $(-35,-29$ and $-23 \mathrm{mV}$ ) lower than $-37 \mathrm{mV}$, the lowest level recorded in the group of normal subjects.

MEASUREMENT UNDER DIRECT VISION

As bile salts increase the permeability of the gastric 


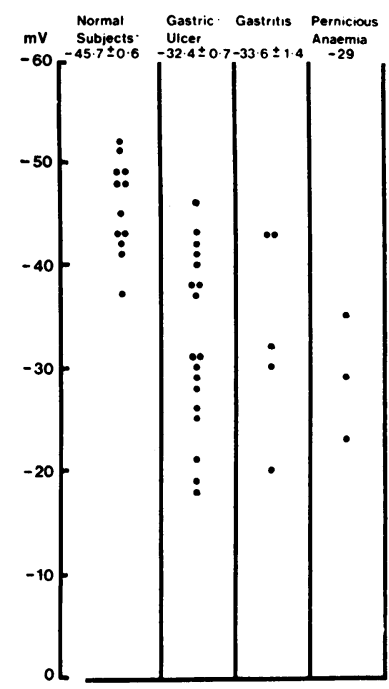

Fig. 1 Potential difference in the body of the stomach in normal volunteers and in three groups of patients. (Mean and SE at top of each column.)

mucosa (Ivey et al., 1970; 1971), and lower the potential difference (Geall et al., 1970), subjects with appreciable amounts of bile-stained fluid in the stomach were placed in a separate group. All four of them had very low potential differences. The mean and SEM for each site are shown in Fig. 2.

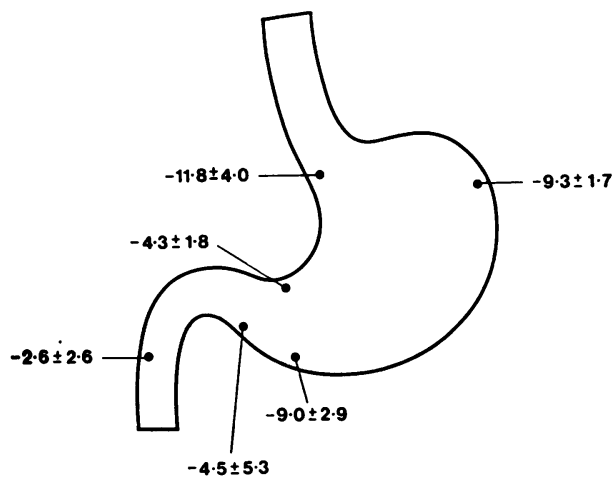

Fig. 2 Potential difference (mean $\pm S E M$ in $m V$ ) in the duodenal bulb and at five sites in the stomach in four subjects with bile reflux

The remaining group of 18 subjects was too small to be divided into sub-groups according to severity of gastritis at various sites. I1 was therefore decided to have only two sub-groups, one with a normal or near-normal mucosa at the high lesser curve, and one with moderate or severe gastritis at that site. In all the patients with gastritis at the high lesser curve, the antrum, and, to a lesser extent, the high greater curve, were also affected.

Twelve subjects had a normal or near-normal mucosa at the high lesser curve. The distribution of the potential difference is shown in Fig. 3. The

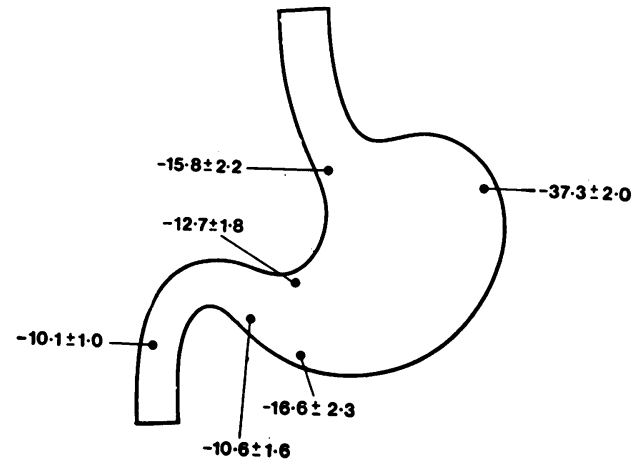

Fig. 3 Potential difference (mean $\pm S E M$ in $m V$ ) in the duodenal bulb and at five sites in the stomach in 12 subjects with normal high lesser curve.

Table 3 Potential difference at high lesser curve and high greater curve in subjects with normal gastric mucosa

\begin{tabular}{lll}
\hline Subject & \multicolumn{2}{l}{ Potential difference $(-m V)$} \\
\cline { 2 - 3 } & High lesser curve & High greater curve \\
\hline 1 & 19 & 45 \\
2 & 15 & 33 \\
3 & 11 & 32 \\
4 & 11 & 38 \\
5 & 9 & 30 \\
6 & 20 & 33 \\
7 & 21 & 41 \\
8 & 22 & 48 \\
9 & 16 & 37 \\
10 & 8 & 32 \\
11 & 32 & 49 \\
12 & 5 & 29 \\
Mean & $15 \cdot 8$ & $37 \cdot 8$ \\
SEM & $\pm 2 \cdot 2$ & $\pm 2 \cdot 0$ \\
\hline
\end{tabular}

individual results for the high lesser curve and the high greater curve are shown in Table 3. The potential difference at the high lesser curve was invariably lower than at the high greater curve.

Six patients had gastritis at the high lesser curve. The distribution of the potential difference is shown in Fig. 4. The mean level at the high lesser curve, $-4.8 \mathrm{mV}$, was significantly $(p<0.01)$ lower than at the same site for subjects without gastritis, where it was $-15 \cdot 8 \mathrm{mV}$.

\section{Discussion}

Hogben (1955) has produced evidence to show that the potential difference across the gastric mucosa of 


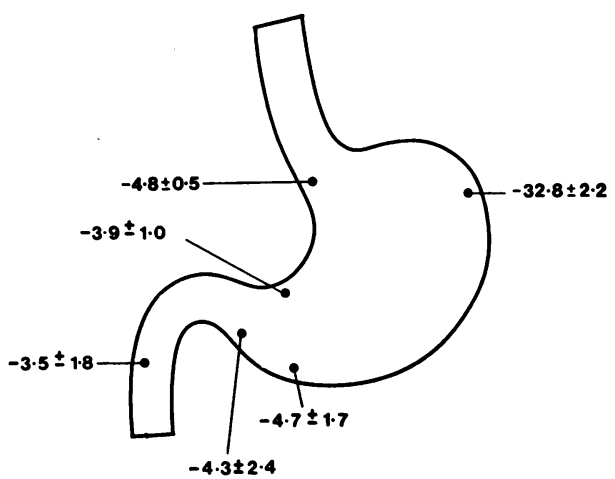

Fig. 4 Potential difference (mean $\pm S E M$ in $m V$ ) in the duodenal bulb and at five sites in the stomach in six subjects with gastritis at the high lesser curve.

the frog is generated by active transport of chloride ions from the serosal to the luminal aspect of the mucosa. The situation is more complex in the mammal, and active transport of sodium and other ions may also be involved (Durbin, 1967). When the mucosa is exposed to an agent that increases its passive ionic permeability, there is a fall in the potential difference that correlates with the increase in ionic fluxes (Davenport et al., 1964; Chvasta and Cooke, 1972). Measurement of ionic fluxes across the gastric mucosa in man is technically difficult, and the assumptions involved in the calculations make results difficult to interpret.

Hence, in spite of the fact that the mechanism of production of the potential difference in man and other mammals is still obscure to a certain extent, the ease with which it can be measured has prompted its use in the study of the effects of drugs on the mucosa (Murray et al., 1974; Hossenbocus and Colin-Jones, 1975). Yet very little has been done to establish normal values in man. Furthermore, although theoretical considerations suggest that the potential difference across the gastritic mucosa should be lower than that across the normal mucosa, the only work on the subject has not been published (quoted by Ivey and Clifton, 1974).

Previous investigations (Andersson and Grossman, 1965; Geall et al., 1970) have shown that the mucosal potential difference is lower in the antrum than in the body of the stomach in normal man. In the present study, the use of the endoscope has made it possible to go further, and to show that the potential difference is low at the high lesser curve as well as in the antrum, and invariably lower there than at the high greater curve. In the dog, the antral mucosa is more permeable than the fundic mucosa (Dyck et al., 1969) and the potential difference is lower in the antrum than in the fundus (Dennis et al., 1959).
Dyck et al. (1969) have suggested that the low antral potential difference in man implies an increased permeability, which may be of importance in view of the high incidence of peptic ulcers in the antrum. The same argument could be applied to the low potential difference found at the high lesser curve here.

Investigators who have measured ionic fluxes in man have found the gastric mucosa to be more permeable in gastric ulcer patients than in normal controls (Chapman et al., 1968, 1972; Ivey and Clifton, 1974). This was so even in those patients in whom the ulcer had healed, suggesting that it was the associated gastritis rather than the ulcer itself that was responsible for the increased permeability. In the present study, whether measurement was made blindly or under direct vision at endoscopy, gastritis was found to be associated with a significantly lower than normal potential difference. It is impossible to say whether the increased passive ionic permeability is the only factor responsible for the lower potential difference found in those patients. The evidence available does not allow one to speculate on the possible role of other factors, such as impairment of the electrogenic transport mechanism.

Nevertheless, if the potential difference is to be used as a tool in clinical research, it is important to be aware of its anatomical distribution, and to realise that it is lower than normal in subjects with gastritis or gastric atrophy.

We wish to thank Mr. P. Salem and Mrs. B. Green for skilled technical assistance; Dr. J. Bamforth for allowing us to study some patients under his care and for his advice. We are also grateful to the Sister and staff of the Metabolic Ward where these studies were carried out.

\section{References}

Andersson, S., and Grossman, M. I. (1965). Profile of pH, pressure, and potential difference at gastroduodenal junction in man. Gastroenterology, 49, 364-371.

Bennet, P. N., Frigo, G. M., Weerasinghe, W. M. T., and Lennard-Jones, J. E. (1975). Effects of carbenoxolone sodium and of aspirin on gastric mucosal potential difference in normal subjects. Scandinavian Journal of Gastroenterology, 10, 161-164.

Chapman, M. L., Werther, J. L., and Janowitz, H. D. (1968). Response of the normal and pathological human gastric mucosa to an instilled acid load. Gastroenterology, 55, 344-353.

Chapman, M. L., Werther, J. L., Rudick, J., and Janowitz, H. D. (1972). Pentagastrin infusion-glycine instillation as a measure of acid absorption in the human stomach: comparison to an instilled acid load. Gastroenterology, 63. 962-972.

Chvasta, T. E., and Cooke, A. R. (1972). The effect of several ulcerogenic drugs on the canine gastric mucosal barrier. Journal of Laboratory and Clinical Medicine, 79, 302-315. 
Davenport, H. W. (1965). Is the apparent hyposecretion of acid by patients with gastric ulcer a consequence of a broken barrier to diffusion of hydrogen ions into the gastric mucosa? Gut, 6, 513 .

Davenport, H. W., Warner, H. A., and Code, C. F. (1964). Functional significance of gastric mucosal barrier to sodium. Gastroenterology, 47, 142-152.

Dennis, W. H., Canosa, C., and Rehm, W. S. (1959). Potential difference across the pyloric antrum. American Journal of Physiology, 197, 19-21.

Department of Health and Social Security (1969). Safety Code for Electro-medical Apparatus. (Hospital Technical Memorandum 8). HMSO: London.

Durbin, R. P. (1967). Electrical potential difference of the gastric mucosa. In Handbook of Physiology, Sect. 6, Alimentary Canal. Edited by C. F. Code, Vol. 2. pp. 879-888. American Physiological Society: Washington DC.

Dyck, W. P., Werther, J. L., Rudick, J., and Janowitz, H. D. (1969). Electrolyte movement across canine antral and fundic gastric mucosa. Gastroenterology, 56, 488-495.

Geall, M. G., Phillips, S. F., and Summerskill, W. H. J. (1970). Profile of gastric potential difference in man. Gastroenterology, 58, 437-443.

Hogben, C. A. M. (1955). Active transport of chloride by isolated frog gastric epithelium: origin of the gastric mucosal potential. American Journal of Physiology, 180, 641-649.

Hossenbocus, A., and Colin-Jones, D. G. (1975). Protection of the human gastric mucosa from aspirin by carbenoxolone. In Fourth Symposium on Carbenoxolone. pp. 91-102. Edited by F. Avery Jones, and D. V. Parke, Butterworths: London.

Ivey, K. J., and Clifton, J. A. (1974). Back diffusion of hydrogen ions across gastric mucosa of patients with gastric ulcer and rheumatoid arthritis. British Medical Journal, 1, 16-19.

Ivey, K. J., DenBesten, L., and Clifton, J. A. (1970). Effect of bile salts on ionic movement across the human gastric mucosa. Gastroenterology, 59, 683-690.

Ivey, K. J., DenBesten, L., and Clifton, J. A. (1971). Effect of intragastric bile salts on ionic movement across normal human gastric mucosa after intravenous atropine. Gut, 12, 257-261.

Murray, H. S., Strottman, M. P., and Cooke, A. R. (1974). Effect of several drugs on gastric potential difference in man. British Medical Journal, 1, 19-21.

Smith, B. M., Skillman, J. J., Edwards, B. G., and Silen, W. (1971). Permeability of the human gastric mucosa: alteration by acetylsalicylic acid and ethanol. New England Medical Journal, 285, 716-721.

Whitehead, R. (1973). Mucosal Biopsy of the Gastro-intestinal Tract, pp. 7-61. Saunders: London.

Whitehead, R., Truelove, S. C., and Gear, W. H. (1972). The histological diagnosis of chronic gastritis in fibreoptic gastroscope biopsy specimens. Journal of Clinical Pathology, 25, 1-11. 